\title{
THE RESEARCH JOURNAL TRANSPORT: PEER-REVIEWING PROCESS IN 2016
}

\author{
Olegas Prentkovskis ${ }^{1}$, Raimundas Junevičius ${ }^{2}$ \\ ${ }^{1}$ Editor-in-Chief of the Research Journal TRANSPORT, E-mail: olegas.prentkovskis@vgtu.lt \\ ${ }^{2}$ Managing (Associate) Editor of the Research Journal TRANSPORT, E-mail: raimundas.junevicius@vgtu.lt
}

The research journal TRANSPORT is published by Vilnius Gediminas Technical University

in partnership with the Lithuanian Academy of Science and Taylor \& Francis

\section{December 2016}

TRANSPORT is a peer-reviewed research journal. To peer-review manuscript submissions, the research journal TRANSPORT uses the interactive system ScholarOne Manuscripts (formerly known as Manuscript Central, http://mc.manuscriptcentral.com/stra).

All manuscripts submitted through ScholarOne Manuscripts are peer-reviewed by members of the Editorial Board or appointed experts. The minimum number of score sheets (peer-review forms filled by reviewers for each submitted manuscript) amounts to three and the average number of score sheets (peer-review forms) is 3.7 in 2016.

Decision ratios of submitted manuscripts in 2011-2016 (source: TRANSPORT ScholarOne Manuscripts, data until 15 December 2016) are presented on Fig. 1. Decision ratios of submitted manuscripts in 2016 (source: TRANSPORT ScholarOne Manuscripts, data until 16 December 2016) are presented on Fig. 2.
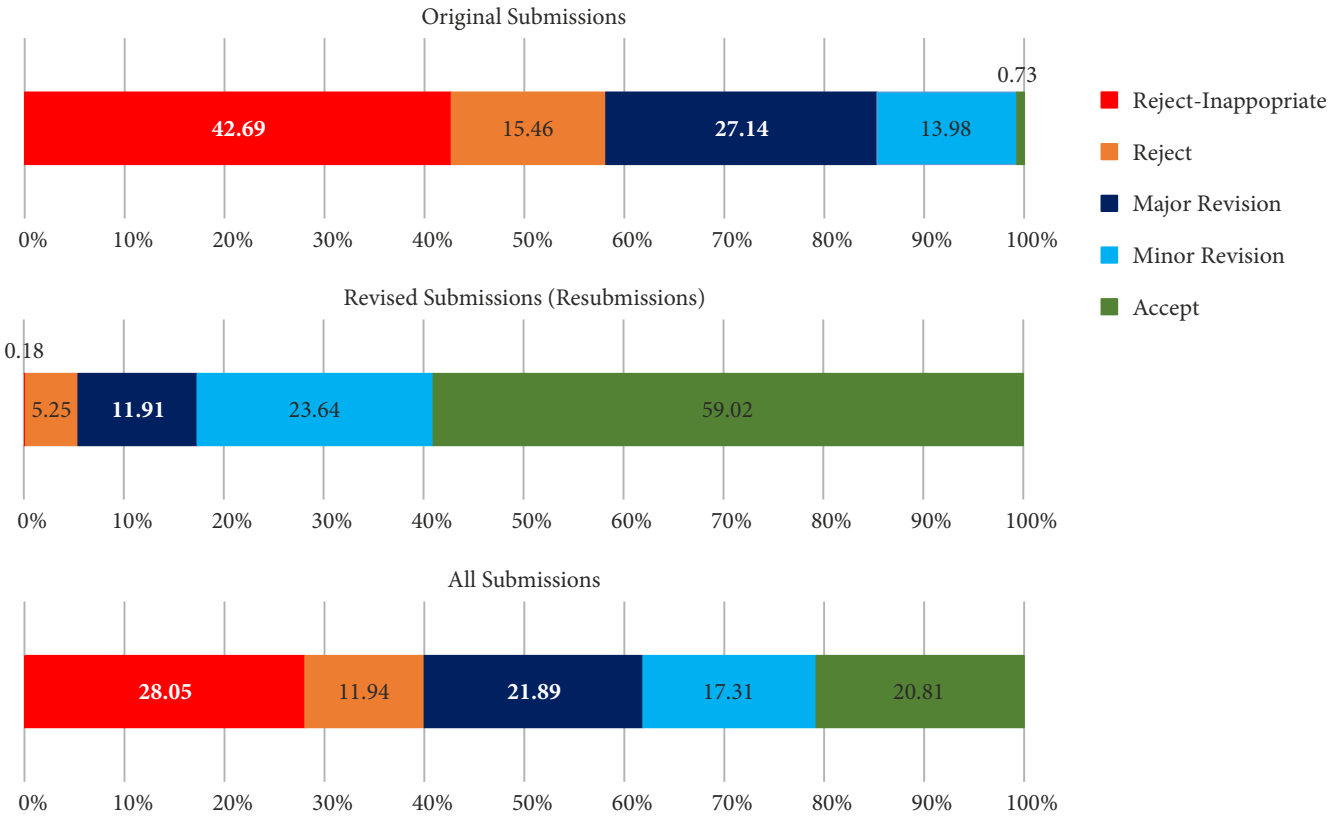

Fig. 1. Decision ratios of submitted manuscripts in 2011-2016

(note: 'revised submissions' follows 'minor revision' or 'major revision' after 'original submissions')

This article has been corrected since first published. Please see the statement of correct (https://doi.org/10.3846/16484142.2017.1284303).

Corresponding author: Olegas Prentkovskis

E-mail: olegas.prentkovskis@vgtu.lt

Copyright @ 2016 Vilnius Gediminas Technical University (VGTU) Press

http://www.tandfonline.com/TRAN 


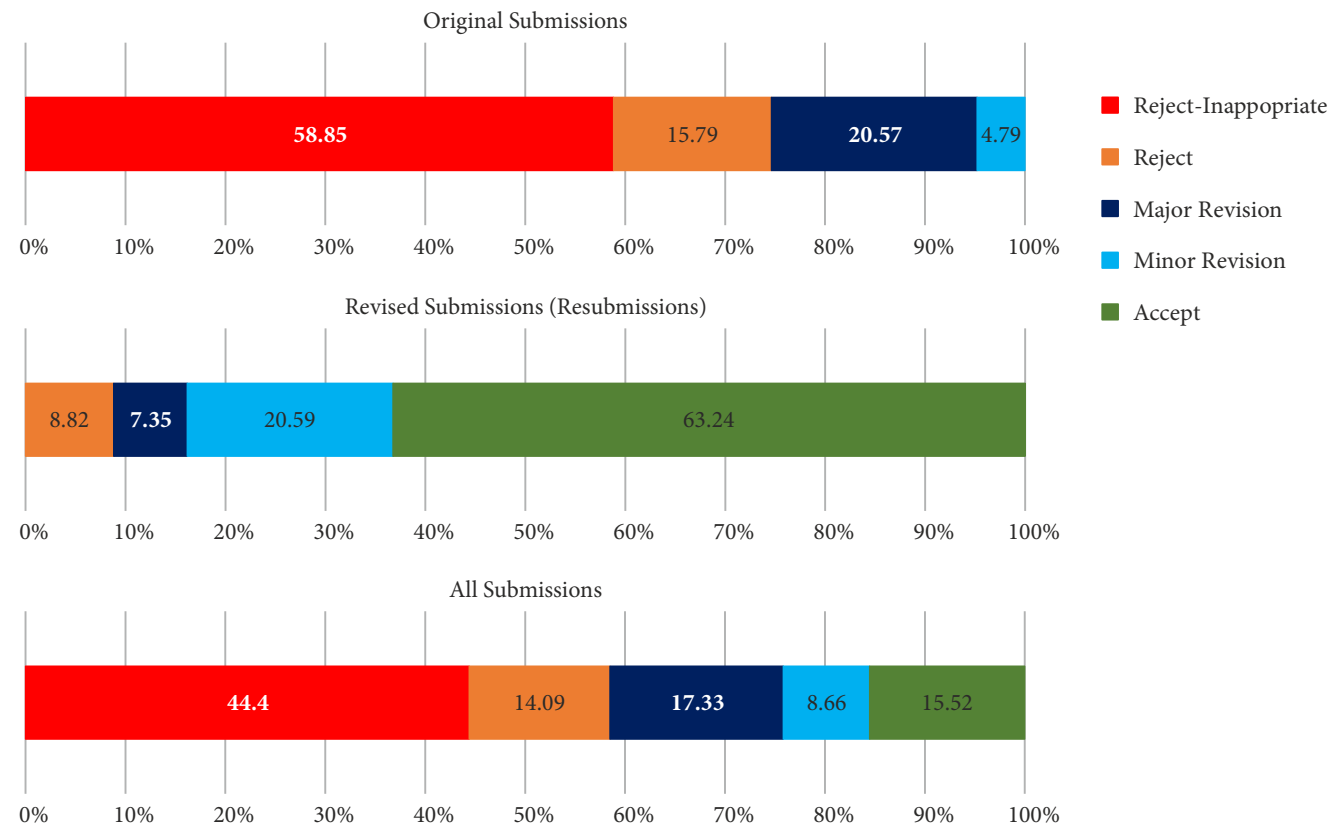

Fig. 2. Decision ratios of submitted manuscripts in 2016

(note: 'revised submissions' follows 'minor revision' or 'major revision' after 'original submissions'

Success and quality of the research journal TRANSPORT depends to a large extent on the activity and performance of its Editorial Board and Reviewers. Reviewers are scientists who volunteer to contribute to the improvement of the quality of transport engineering and transport management fields by guiding authors in their efforts to publish their research outputs that help to enlarge the scientific content of the research journal TRANSPORT, for which we feel truly grateful.

Thus, on behalf of the Editorial Board of the research journal TRANSPORT we would like to express our gratitude to the following reviewers (Table) for their important contribution to the quality of scientific articles submitted in 2013-2016 (accepted in 2014-2016), which were peer-reviewed and part of them - published in TRANSPORT $31(1,2,3,4) 2016$ or as 'Article in Press' (Latest Articles on www).

Table. List of reviewers of scientific peer-reviewed articles submitted in 2013-2016 (accepted in 2014-2016 and published in TRANSPORT 31(1, 2, 3, 4) 2016 or as 'Article in Press')

\begin{tabular}{|l|l|}
\hline \multicolumn{1}{|c|}{ Surname, Name } & \multicolumn{1}{c|}{ Institution } \\
\hline Spiryagin, Maksym & Central Queensland University \\
\hline Yarlagadda, Prasad & $\begin{array}{l}\text { Queensland University } \\
\text { of Technology }\end{array}$ \\
\cline { 1 - 1 } Hutchinson, Paul & University of Adelaide \\
\cline { 1 - 1 } Bambach, Mike & University of New South Wales \\
\cline { 1 - 1 } Assemi, Behrang & \multirow{2}{*}{ University of Queensland } \\
\cline { 1 - 1 } Prato, Carlo & \multirow{2}{*}{ University of South Australia } \\
\cline { 1 - 1 } Tavassoli, Ahmad & \multirow{2}{*}{ University of Sydney } \\
\cline { 1 - 1 } Taylor, Michael & \\
\cline { 1 - 1 } Chung, Demi &
\end{tabular}

\begin{tabular}{|l|l|}
\hline \multicolumn{1}{|c|}{ Surname, Name } & \multicolumn{1}{c|}{ Institution } \\
\cline { 1 - 1 } Mulley, Corinne & \multirow{2}{*}{ University of Sydney } \\
\cline { 1 - 1 } Oesterle, Ines & Austria \\
\cline { 1 - 1 } Stopher, Peter & $\begin{array}{l}\text { University of Applied Sciences } \\
\text { Upper Austria }\end{array}$ \\
\cline { 1 - 1 } Lindorfer, Manuel & $\begin{array}{l}\text { Vienna University } \\
\text { of Technology }\end{array}$ \\
\cline { 1 - 1 } Edelmann, Johannes & \\
\cline { 1 - 1 } Kopacek, Peter & Belgium \\
\hline Schulz, Alexander & Free University of Brussels \\
\hline Janjevic, Milena & University of Liège \\
\hline Verlinde, Sara &
\end{tabular}




\begin{tabular}{|c|c|}
\hline Surname, Name & Institution \\
\hline Fei, Hongying & \multirow[t]{3}{*}{ University of Mons } \\
\hline Kouroussis, Georges & \\
\hline Verlinden, Olivier & \\
\hline Macharis, Cathy & Vrije Universiteit Brussel \\
\hline \multicolumn{2}{|r|}{ Brazil } \\
\hline Carlson, Rodrigo & $\begin{array}{l}\text { Federal University of Santa } \\
\text { Catarina }\end{array}$ \\
\hline $\begin{array}{l}\text { Gongora-Rubio, Mário } \\
\text { Ricardo }\end{array}$ & $\begin{array}{l}\text { Institute for Technological } \\
\text { Research }\end{array}$ \\
\hline Morooka, Celso & State University of Campinas \\
\hline \multicolumn{2}{|r|}{ Canada } \\
\hline Alecsandru, Ciprian & Concordia University \\
\hline El-Geneidy, Ahmed & McGill University \\
\hline Huang, Kai & McMaster University \\
\hline Trépanier, Martin & Polytechnique de Montréal \\
\hline Reed, Maureen & Ryerson University \\
\hline Yin, Derek & University of Alberta \\
\hline Kabir, Golam & University of British Columbia \\
\hline Kattan, Lina & University of Calgary \\
\hline $\mathrm{Ng}$, Adolf & University of Manitoba \\
\hline Younes, Maram Bani & University of Ottawa \\
\hline Dai, Li Ming & University of Regina \\
\hline Bachmann, Chris & University of Toronto \\
\hline Lee, Chris & University of Windsor \\
\hline \multicolumn{2}{|r|}{ China } \\
\hline Ma, Weijia & $\begin{array}{l}\text { AVIC Aerodynamics Research } \\
\text { Institute }\end{array}$ \\
\hline Li, Hongqi & Beihang University \\
\hline Bin, Xin & Beijing Institute of Technology \\
\hline Chen, Shaokuan & \multirow[t]{2}{*}{ Beijing Jiaotong University } \\
\hline Gao, Liang & \\
\hline Zhao, Xiaohua & $\begin{array}{l}\text { Beijing University } \\
\text { of Technology }\end{array}$ \\
\hline Peng, Yong & Central South University \\
\hline Wang, Yonggang & Chang'an University \\
\hline Zhang, Zhichao & $\begin{array}{l}\text { China Academy of Railway } \\
\text { Sciences }\end{array}$ \\
\hline Wang, Tianxing & Chinese Academy of Sciences \\
\hline Lu, Jing & \multirow[t]{3}{*}{ Dalian Maritime University } \\
\hline Yu, Bin & \\
\hline Zeng, Qingcheng & \\
\hline
\end{tabular}

\begin{tabular}{|c|c|}
\hline Surname, Name & Institution \\
\hline $\mathrm{Xu}$, Hongfeng & \multirow{2}{*}{$\begin{array}{l}\text { Dalian University } \\
\text { of Technology }\end{array}$} \\
\hline Zhang, Lihui & \\
\hline Gu, Guangxin & Fudan University \\
\hline Bie, Yiming & Harbin Institute of Technology \\
\hline Hou, Shujuan & Hunan University \\
\hline Yang, Xinfeng & Lanzhou Jiaotong University \\
\hline Lu, Ai-Zhong & $\begin{array}{l}\text { North China Electric Power } \\
\text { University }\end{array}$ \\
\hline Cai, Zhiqiang & \multirow{2}{*}{$\begin{array}{l}\text { Northwestern Polytechnical } \\
\text { University }\end{array}$} \\
\hline Chen, Jian-Zhong & \\
\hline Liu, Yu & Peking University \\
\hline An, Xiaomi & Renmin University of China \\
\hline Ge, Ying-En & Shanghai Maritime University \\
\hline Wei, Gui-wu & Sichuan Normal University \\
\hline Cheng, Jian-Chuan & \multirow[t]{3}{*}{ Southeast University } \\
\hline Li, Zhibin & \\
\hline Wang, Weize & \\
\hline Jin, Xuesong & \multirow[t]{2}{*}{ Southwest Jiaotong University } \\
\hline Zhai, Wanming & \\
\hline Lun, Zhang & \multirow[t]{2}{*}{ Tongji University } \\
\hline Zhang, Xiaoning & \\
\hline \multicolumn{2}{|c|}{ Colombia } \\
\hline $\begin{array}{l}\text { Arango Serna, Martin } \\
\text { Dario }\end{array}$ & $\begin{array}{l}\text { National University } \\
\text { of Colombia }\end{array}$ \\
\hline \multicolumn{2}{|r|}{ Croatia } \\
\hline Pupavac, Drago & Polytechnic of Rijeka \\
\hline Krile, Srecko & University of Dubrovnik \\
\hline $\begin{array}{l}\text { Deluka-Tibljaš, } \\
\text { Aleksandra }\end{array}$ & \multirow[t]{4}{*}{ University of Rijeka } \\
\hline Hess, Mirano & \\
\hline Hess, Svjetlana & \\
\hline Tijan, Edvard & \\
\hline Breski, Deana & \multirow[t]{2}{*}{ University of Split } \\
\hline Cvitanić, Dražen & \\
\hline Anžek, Mario & \multirow[t]{6}{*}{ University of Zagreb } \\
\hline Babić, Darko & \\
\hline Barić, Danijela & \\
\hline Ćavar, Ivana & \\
\hline Peraković, Dragan & \\
\hline Sostaric, Marko & \\
\hline
\end{tabular}




\begin{tabular}{|c|c|}
\hline Surname, Name & Institution \\
\hline \multicolumn{2}{|c|}{ Czech Republic } \\
\hline Chlebek, Jiří & \multirow[t]{4}{*}{ Brno University of Technology } \\
\hline Daněk, Vladimír & \\
\hline Porteš, Petr & \\
\hline Vosecký, Slavomír & \\
\hline Baudyš, Karel & \multirow{8}{*}{$\begin{array}{l}\text { Czech Technical University } \\
\text { in Prague }\end{array}$} \\
\hline Beneš, Libor & \\
\hline Bína, Ladislav & \\
\hline Čarská, Zuzana & \\
\hline Janoš, Vít & \\
\hline Kocourek, Josef & \\
\hline Kočárková, Dagmar & \\
\hline Neubergová, Kristýna & \\
\hline Brabec, Marek & $\begin{array}{l}\text { Institute of Compuer Science, } \\
\text { Academy of Sciences of th } \\
\text { Czech Republic }\end{array}$ \\
\hline Kolar, Vaclav & \multirow[t]{2}{*}{ Technical University of Ostrava } \\
\hline Stýskala, Vítězslav & \\
\hline Stopka, Ondrej & $\begin{array}{l}\text { Institute of Technology and } \\
\text { Business in České Budějovice }\end{array}$ \\
\hline Soukopova, Jana & Masaryk University \\
\hline Černý, Jan & $\begin{array}{l}\text { University of Economics } \\
\text { in Prague }\end{array}$ \\
\hline Bulíček, Josef & \multirow[t]{6}{*}{ University of Pardubice } \\
\hline Drahotský, Ivo & \\
\hline Lata, Michael & \\
\hline Matuška, Jaroslav & \\
\hline Hruška, Roman & \\
\hline Soušek, Radovan & \\
\hline Kraft, Stanislav & $\begin{array}{l}\text { University of South Bohemia } \\
\text { in České Budějovice }\end{array}$ \\
\hline Gottvald, Jakub & VÍTKOVICE ÚAM, Inc. \\
\hline \multicolumn{2}{|c|}{ Denmark } \\
\hline Anderson, Marie & \multirow{7}{*}{$\begin{array}{l}\text { Technical University } \\
\text { of Denmark }\end{array}$} \\
\hline Barfod, Michael & \\
\hline Gudmundsson, Henrik & \\
\hline Kaplan, Sigal & \\
\hline Mulalic, Ismir & \\
\hline Nielsen, Otto & \\
\hline Rasmussen, Thomas & \\
\hline
\end{tabular}

\begin{tabular}{|c|c|}
\hline Surname, Name & Institution \\
\hline \multicolumn{2}{|c|}{ Ecuador } \\
\hline $\begin{array}{l}\text { Macías Párraga, Mariela } \\
\text { Jahaira }\end{array}$ & $\begin{array}{l}\text { Ministry of Transport and } \\
\text { Public Works of the Ecuador }\end{array}$ \\
\hline \multicolumn{2}{|r|}{ Estonia } \\
\hline Segercrantz, Wladimir & $\begin{array}{l}\text { Tallinn University of Applied } \\
\text { Sciences }\end{array}$ \\
\hline Aavik, Andrus & \multirow{2}{*}{$\begin{array}{l}\text { Tallinn University } \\
\text { of Technology }\end{array}$} \\
\hline Antov, Dago & \\
\hline \multicolumn{2}{|r|}{ Ethiopia } \\
\hline Beshah, Tibebe & Addis Ababa University \\
\hline \multicolumn{2}{|r|}{ Finland } \\
\hline Bruun, Eric & \multirow[t]{4}{*}{ Aalto University } \\
\hline Matusiak, Jerzy & \\
\hline Montewka, Jakub & \\
\hline Siikonen, Timo & \\
\hline Salminen, Jani & Finnish Environment Institute \\
\hline Kujala, Pentti & $\begin{array}{l}\text { Helsinki University } \\
\text { of Technology }\end{array}$ \\
\hline Liimatainen, Heikki & $\begin{array}{l}\text { Tampere University } \\
\text { of Technology }\end{array}$ \\
\hline Repka, Sari & \multirow[t]{2}{*}{ University of Turku } \\
\hline Tapaninen, Ulla & \\
\hline \multicolumn{2}{|r|}{ France } \\
\hline Herrero, German & Atos S.A. \\
\hline Khoudour, Louahdi & CEREMA \\
\hline Bhouri, Neila & $\begin{array}{l}\text { French Institute of Science } \\
\text { and Technology for Transport, } \\
\text { Spatial Planning, Development } \\
\text { and Networks - IFSTTAR }\end{array}$ \\
\hline Gonzalez-Feliu, Jesus & Mines School of Saint-Étienne \\
\hline \multicolumn{2}{|r|}{ Germany } \\
\hline Amancio, Sergio & $\begin{array}{l}\text { Centre for Materials an Coastal } \\
\text { Research }\end{array}$ \\
\hline Heinitz, Florian & $\begin{array}{l}\text { Erfurt University of Applied } \\
\text { Science }\end{array}$ \\
\hline Ivanov, Valentin & \begin{tabular}{|l|} 
Ilmenau University \\
of Technology
\end{tabular} \\
\hline Acciaro, Michele & Kühne Logistics University \\
\hline Krüger, Philip & \multirow{2}{*}{$\begin{array}{l}\text { Technical University } \\
\text { of Darmstadt }\end{array}$} \\
\hline Zhang, Ning & \\
\hline Magyar, Balázs & University of Kaiserslautern \\
\hline \multicolumn{2}{|r|}{ Greece } \\
\hline Giannopoulos, George & \multirow{2}{*}{$\begin{array}{l}\text { Aristotle University } \\
\text { of Thessaloniki }\end{array}$} \\
\hline Samaras, Zisis & \\
\hline
\end{tabular}




\begin{tabular}{|c|c|}
\hline Surname, Name & Institution \\
\hline Bekiaris, Evangelos & \multirow{6}{*}{$\begin{array}{l}\text { Center for Research } \\
\text { and Technology Hellas }\end{array}$} \\
\hline Chaniotakis, Emmanouil & \\
\hline Mintsis, Vagelis & \\
\hline $\begin{array}{l}\text { Salanova Grau, Josep } \\
\text { Maria }\end{array}$ & \\
\hline Stamos, Iraklis & \\
\hline $\begin{array}{l}\text { Stathacopoulos, } \\
\text { Alexander }\end{array}$ & \\
\hline Georgoudas, Ioakeim & $\begin{array}{l}\text { Democritus University } \\
\text { of Thrace }\end{array}$ \\
\hline Loizos, Andreas & \multirow{4}{*}{$\begin{array}{l}\text { National Technical University } \\
\text { of Athens }\end{array}$} \\
\hline Theofilatos, Athanasios & \\
\hline Vlahogianni, Eleni & \\
\hline Yannis, George & \\
\hline $\begin{array}{l}\text { Psomopoulos, } \\
\text { Constantinos }\end{array}$ & $\begin{array}{l}\text { Technological Education } \\
\text { Institute of Piraeus }\end{array}$ \\
\hline Tzannatos, Ernestos & University of Piraeus \\
\hline Nikitakos, Nikitas & \multirow[t]{2}{*}{ University of the Aegean } \\
\hline Pallis, Athanasios & \\
\hline Vogiatzis, Konstantinos & University of Thessaly \\
\hline \multicolumn{2}{|c|}{ Hong Kong } \\
\hline Yuen, Kevin Kam Fung & \multirow{2}{*}{$\begin{array}{l}\text { Hong Kong Polytechnic } \\
\text { University }\end{array}$} \\
\hline Zhu, Wenting & \\
\hline Huang, George & \multirow[t]{2}{*}{ University of Hong Kong } \\
\hline Szeto, Wai Yuen & \\
\hline \multicolumn{2}{|c|}{ Hungary } \\
\hline Denke, Zsolt & $\begin{array}{l}\text { BKK Centre for Budapest } \\
\text { Transport }\end{array}$ \\
\hline Bereczky, Ákos & \multirow{9}{*}{$\begin{array}{l}\text { Budapest University } \\
\text { of Technology and Economics }\end{array}$} \\
\hline Bokor, Zoltan & \\
\hline Csiszár, Csaba & \\
\hline Markovits, Tamás & \\
\hline Péter, Horák & \\
\hline Péter, Tamás & \\
\hline Tanczos, Katalin & \\
\hline Tettamanti, Tamás & \\
\hline Török, Ádám & \\
\hline Duleba, Szabolcs & College of Nyíregyháza \\
\hline Gaspar, Peter & $\begin{array}{l}\text { Computer and Automation } \\
\text { Research Institute }\end{array}$ \\
\hline Markovits-Somogyi, Rita & $\begin{array}{l}\text { HungaroControl - Hungarian } \\
\text { Air Navigation Services Pte. } \\
\text { Ltd. Co. }\end{array}$ \\
\hline
\end{tabular}

\begin{tabular}{|c|c|}
\hline Surname, Name & Institution \\
\hline Zöldy, Máté & MOL DS Product Development \\
\hline \multicolumn{2}{|r|}{ India } \\
\hline Patil, Gopal & \multirow{2}{*}{$\begin{array}{l}\text { Indian Institute of Technology } \\
\text { Bombay }\end{array}$} \\
\hline Singh, Dharamveer & \\
\hline Chunchu, Mallikarjuna & $\begin{array}{l}\text { Indian Institute of Technology } \\
\text { Guwahati }\end{array}$ \\
\hline Pandit, Debapratim & $\begin{array}{l}\text { Indian Institute of Technology } \\
\text { Kharagpur }\end{array}$ \\
\hline Vanajakshi, Lelitha & $\begin{array}{l}\text { Indian Institute of Technology } \\
\text { Madras }\end{array}$ \\
\hline Bhuyan, Prasanta & $\begin{array}{l}\text { National Institute of Technology } \\
\text { Rourkela }\end{array}$ \\
\hline \multicolumn{2}{|r|}{ Iran } \\
\hline Esmaeili, Ahmad & Allameh Tabataba’i University \\
\hline Shafabakhsh, Gholamali & Semnan University \\
\hline Abdi, Javad & University of Tehran \\
\hline \multicolumn{2}{|r|}{ Ireland } \\
\hline Enright, Bernard & Dublin Institute of Technology \\
\hline Chen, I-Shuo & $\begin{array}{l}\text { Trinity College Dublin, the } \\
\text { University of Dublin }\end{array}$ \\
\hline \multicolumn{2}{|r|}{ Israel } \\
\hline Bekhor, Shlomo & \multirow{2}{*}{$\begin{array}{l}\text { Technion - Israel Institute } \\
\text { of Technology }\end{array}$} \\
\hline Gitelman, Victoria & \\
\hline \multicolumn{2}{|r|}{ Italy } \\
\hline Di Pasquale, Guide & Pluservice srl. \\
\hline Bascetta, Luca & Polytechnic University of Milan \\
\hline Pronello, Cristina & Polytechnic University of Turin \\
\hline Cipriani, Ernesto & Roma Tre University \\
\hline Cantisani, Giuseppe & \multirow[t]{2}{*}{ Sapienza University of Rome } \\
\hline Loprencipe, Giuseppe & \\
\hline Bortolini, Marco & \multirow[t]{4}{*}{ University of Bologna } \\
\hline Molari, Giovanni & \\
\hline Simone, Andrea & \\
\hline Vignali, Valeria & \\
\hline Pani, Claudia & University of Cagliari \\
\hline Mazzulla, Gabriella & University of Calabria \\
\hline D’Apuzzo, Mauro & University of Cassino \\
\hline Meli, Enrico & University of Florence \\
\hline Pellegrino, Orazio & University of Messina \\
\hline Hadjidimitriou, Selini & \multirow{2}{*}{$\begin{array}{l}\text { University of Modena and } \\
\text { Reggio Emilia }\end{array}$} \\
\hline Novellani, Stefano & \\
\hline
\end{tabular}




\begin{tabular}{|c|c|}
\hline Surname, Name & Institution \\
\hline Abbondati, Francesco & \multirow[t]{6}{*}{ University of Naples Federico II } \\
\hline $\begin{array}{l}\text { Biancardo, Salvatore } \\
\text { Antonio }\end{array}$ & \\
\hline Busiello, Mariarosaria & \\
\hline De Luca, Mario & \\
\hline Dell‘Acqua, Gianluca & \\
\hline Russo, Francesca & \\
\hline Granà, Anna & University of Palermo \\
\hline Giuliani, Felice & University of Parma \\
\hline Nicolosi, Vittorio & University of Rome Tor Vergata \\
\hline Mauro, Raffaele & University of Trento \\
\hline Danielis, Romeo & \multirow[t]{3}{*}{ University of Trieste } \\
\hline Francescutto, Alberto & \\
\hline Ukovich, Walter & \\
\hline \multicolumn{2}{|r|}{ Japan } \\
\hline Munehiro, Kazunori & $\begin{array}{l}\text { Civil Engimeering Research } \\
\text { Institute for Cold Region }\end{array}$ \\
\hline Uchida, Kenetsu & Hokkaido University \\
\hline Li, Guoquan & $\begin{array}{l}\text { Railway Technical Research } \\
\text { Institute }\end{array}$ \\
\hline Mishina, Tsutomu & Seibi University Junior College \\
\hline Paul, Topon & Toshiba Corporation \\
\hline \multicolumn{2}{|r|}{ Jordan } \\
\hline Al-Omari, Bashar & $\begin{array}{l}\text { Jordan University of Science } \\
\text { and Technology }\end{array}$ \\
\hline \multicolumn{2}{|r|}{ Korea } \\
\hline Park, Simsoo & Korea University \\
\hline Kim, Seon Jin & Pukyong National University \\
\hline Kim, Daejin & \multirow[t]{2}{*}{ Seoul Institute } \\
\hline Ko, Joonho & \\
\hline Lee, Seungjae & University of Seoul \\
\hline \multicolumn{2}{|r|}{ Latvia } \\
\hline Grīslis, Aivis & \multirow[t]{6}{*}{ Riga Technical University } \\
\hline Karevs, Vladimirs & \\
\hline Mezītis, Mareks & \\
\hline Mor-Yaroslavtsev, Andrejs & \\
\hline Smirnovs, Juris & \\
\hline Vība, Jānis & \\
\hline Kabashkin, Igor & \multirow{2}{*}{$\begin{array}{l}\text { Transport and } \\
\text { Telecommunication Institute }\end{array}$} \\
\hline Yatskiv (Jackiva), Irina & \\
\hline
\end{tabular}

\begin{tabular}{|c|c|}
\hline Surname, Name & Institution \\
\hline \multicolumn{2}{|c|}{ Lithuania } \\
\hline Makarevičienè, Violeta & \multirow{2}{*}{$\begin{array}{l}\text { Aleksandras Stulginskis } \\
\text { University }\end{array}$} \\
\hline Žunda, Audrius & \\
\hline Vitkūnas, Rolandas & $\begin{array}{l}\text { International School of Law } \\
\text { and Business }\end{array}$ \\
\hline Dailydka, Stasys & \multirow[t]{2}{*}{ JSC 'Lithuanian Railways' } \\
\hline Jarašūnienè, Aldona & \\
\hline Bazaras, Žilvinas & \multirow{15}{*}{$\begin{array}{l}\text { Kaunas University } \\
\text { of Technology }\end{array}$} \\
\hline Fedaravičius, Algimantas & \\
\hline Gargasas, Vytautas & \\
\hline Griškevičius, Paulius & \\
\hline $\begin{array}{l}\text { Ilgakojytè-Bazariené, } \\
\text { Jurga }\end{array}$ & \\
\hline Jablonskytė, Janina & \\
\hline $\begin{array}{l}\text { Kandrotaité-Janutiené, } \\
\text { Rasa }\end{array}$ & \\
\hline Keršys, Artūras & \\
\hline Keršys, Robertas & \\
\hline Kliučinskas, Linas & \\
\hline Makaras, Rolandas & \\
\hline Martuzevičius, Dainius & \\
\hline Pranevičius, Henrikas & \\
\hline Raslavičius, Laurencas & \\
\hline Skvireckas, Ramūnas & \\
\hline Andziulis, Arūnas & \multirow[t]{6}{*}{ Klaipèda University } \\
\hline Janutėnienè, Jolanta & \\
\hline Lebedevas, Sergejus & \\
\hline Mažeika, Pranas & \\
\hline Paulauskas, Vytautas & \\
\hline Smailys, Vytautas & \\
\hline Rudzkiené, Vitalija & Mykolas Romeris University \\
\hline Jakubauskas, Gražvydas & \multirow{2}{*}{$\begin{array}{l}\text { Ministry of Transport and } \\
\text { Communications of the } \\
\text { Rebuplic of Lithuania }\end{array}$} \\
\hline Pumputis, Vidmantas & \\
\hline Antuchevičienė, Jurgita & \multirow{8}{*}{$\begin{array}{l}\text { Vilnius Gediminas } \\
\text { Technical University }\end{array}$} \\
\hline Barysienè, Jurgita & \\
\hline Baublys, Adolfas & \\
\hline Batarliené, Nijolè & \\
\hline Bazaras, Darius & \\
\hline Bogdevičius, Marijonas & \\
\hline Braziūnas, Justas & \\
\hline Bručas, Domantas & \\
\hline
\end{tabular}




\begin{tabular}{|c|c|}
\hline Surname, Name & Institution \\
\hline Bučinskas, Vytautas & \multirow{38}{*}{$\begin{array}{l}\text { Vilnius Gediminas } \\
\text { Technical University }\end{array}$} \\
\hline Bureika, Gintautas & \\
\hline Burinskienė, Marija & \\
\hline Čižiūnienė, Kristina & \\
\hline Davidavičienè, Vida & \\
\hline Gribniak, Viktor & \\
\hline Garbinčius, Giedrius & \\
\hline Grigonis, Vytautas & \\
\hline Griškevičius, Julius & \\
\hline Jakimavičius, Marius & \\
\hline Junevičius, Raimundas & \\
\hline Kondroška, Vaidotas & \\
\hline Liudvinavičius, Lionginas & \\
\hline $\begin{array}{l}\text { Meidutè-Kavaliauskienè, } \\
\text { Ieva }\end{array}$ & \\
\hline Miškinis, Paulius & \\
\hline Nagurnas, Saulius & \\
\hline Oginskas, Rolandas & \\
\hline Palšaitis, Ramūnas & \\
\hline Pečeliūnas, Robertas & \\
\hline Podvezko, Valentinas & \\
\hline Prentkovskis, Olegas & \\
\hline Pukalskas, Saugirdas & \\
\hline Rimkus, Alfredas & \\
\hline Rudinskas, Darius & \\
\hline Sivilevičius, Henrikas & \\
\hline Skrickij, Viktor & \\
\hline Sokolovskij, Edgar & \\
\hline $\begin{array}{l}\text { Sužiedelytè-Visockienė, } \\
\text { Jūratė }\end{array}$ & \\
\hline Turskis, Zenonas & \\
\hline Udris, Dainius & \\
\hline $\begin{array}{l}\text { Ušpalytė-Vitkūnienė, } \\
\text { Rasa }\end{array}$ & \\
\hline Vadluga, Vaidas & \\
\hline Vaičiūnas, Gediminas & \\
\hline Vaidogas, Egidijus & \\
\hline Vaišis, Vaidotas & \\
\hline Vaiškūnaitè, Rasa & \\
\hline Vaitkus, Audrius & \\
\hline Valivonis, Juozas & \\
\hline
\end{tabular}

\begin{tabular}{|c|c|}
\hline Surname, Name & Institution \\
\hline Vasarevičius, Saulius & \multirow{8}{*}{$\begin{array}{l}\text { Vilnius Gediminas } \\
\text { Technical University }\end{array}$} \\
\hline $\begin{array}{l}\text { Vasilienè-Vasiliauskienè, } \\
\text { Virgilija }\end{array}$ & \\
\hline Vasilis Vasiliauskas, Aidas & \\
\hline Vekteris, Vladas & \\
\hline Vorobjovas, Viktoras & \\
\hline Zavadskas, Edmundas & \\
\hline Žilionienè, Daiva & \\
\hline Žuraulis, Vidas & \\
\hline Štreimikienė, Dalia & \multirow[t]{2}{*}{ Vilnius University } \\
\hline Veteikis, Darijus & \\
\hline \multicolumn{2}{|c|}{ Malaysia } \\
\hline Gunawan, Teddy & $\begin{array}{l}\text { International Islamic University } \\
\text { Malaysia }\end{array}$ \\
\hline Khoo, Hooi Ling & $\begin{array}{l}\text { Tunku Abdul Rahman } \\
\text { University }\end{array}$ \\
\hline Ahmad, Mohd & University of Malaysia Pahang \\
\hline \multicolumn{2}{|c|}{ Mexico } \\
\hline $\begin{array}{l}\text { Cedillo-Campos, Miguel } \\
\text { Gaston }\end{array}$ & Mexican Institute of Transport \\
\hline Cedillo, Gaston & $\begin{array}{l}\text { Mexican Logistics and Supply } \\
\text { Chain Association }\end{array}$ \\
\hline Lárraga, María & $\begin{array}{l}\text { National Autonomous } \\
\text { University of Mexico }\end{array}$ \\
\hline \multicolumn{2}{|c|}{ Montenegro } \\
\hline Bauk, Sanja & \multirow[t]{2}{*}{ University of Montenegro } \\
\hline Dragović, Branislav & \\
\hline \multicolumn{2}{|c|}{ Netherlands } \\
\hline Liao, Feixiong & $\begin{array}{l}\text { Eindhoven University } \\
\text { of Technology }\end{array}$ \\
\hline \multicolumn{2}{|c|}{ Norway } \\
\hline Klein-Paste, Alex & $\begin{array}{l}\text { Norwegian University } \\
\text { of Science and Technology }\end{array}$ \\
\hline Starykov, Maksym & Palfinger Marine Norway AS \\
\hline \multicolumn{2}{|c|}{ Pakistan } \\
\hline Abbas, Mazhar & University of Gujrat \\
\hline \multicolumn{2}{|c|}{ Panama } \\
\hline Quijada Alarcón, Jorge & $\begin{array}{l}\text { Technological University } \\
\text { of Panama }\end{array}$ \\
\hline \multicolumn{2}{|c|}{ Poland } \\
\hline Szpica, Dariusz & $\begin{array}{l}\text { Bialystok University } \\
\text { of Technology }\end{array}$ \\
\hline Tracz, Marian & $\begin{array}{l}\text { Tadeusz Kościuszko Cracow } \\
\text { University of Technology }\end{array}$ \\
\hline
\end{tabular}




\begin{tabular}{|c|c|}
\hline Surname, Name & Institution \\
\hline Bartłomiejczyk, Mikołaj & $\begin{array}{l}\text { Gdansk University } \\
\text { of Technology }\end{array}$ \\
\hline Potkański, Wojciech & Institute of Aviation \\
\hline Borkowski, Piotr & Lodz University of Technology \\
\hline Chernets, Myron & \multirow[t]{2}{*}{ Lublin University of Technology } \\
\hline Koszałka, Grzegorz & \\
\hline Nowacki, Gabriel & \multirow{2}{*}{$\begin{array}{l}\text { Military University } \\
\text { of Technology }\end{array}$} \\
\hline Prochowski, Leon & \\
\hline Kossecki, Pawel & $\begin{array}{l}\text { Leon Schiller National Higher } \\
\text { School of Film, Television and } \\
\text { Theatre }\end{array}$ \\
\hline Paslawski, Jerzy & \multirow{2}{*}{$\begin{array}{l}\text { Poznan University } \\
\text { of Technology }\end{array}$} \\
\hline Sawicki, Piotr & \\
\hline Sybilski, Dariusz & $\begin{array}{l}\text { Road and Bridge Research } \\
\text { Institute }\end{array}$ \\
\hline Krawiec, Stanisław & \multirow{3}{*}{$\begin{array}{l}\text { Silesian University } \\
\text { of Technology }\end{array}$} \\
\hline Sitarz, Marek & \\
\hline Sładkowski, Aleksander & \\
\hline Bąk, Monika & \multirow[t]{2}{*}{ University of Gdańsk } \\
\hline Dąbrowski, Janusz & \\
\hline Załoga, Elżbieta & University of Szczecin \\
\hline Wierzbicki, Slawomir & $\begin{array}{l}\text { University of Warmia and } \\
\text { Mazury in Olsztyn }\end{array}$ \\
\hline Witkowski, Jaroslaw & $\begin{array}{l}\text { Wrocław University } \\
\text { of Economics }\end{array}$ \\
\hline Golonka, Leszek & $\begin{array}{l}\text { Wrocław University of Science } \\
\text { and Technology }\end{array}$ \\
\hline \multicolumn{2}{|c|}{ Portugal } \\
\hline Marques, Rui Cunha & Technical University of Lisbon \\
\hline Tchepel, Oxana & University of Aveiro \\
\hline Bastos Silva, Ana & \multirow[t]{3}{*}{ University of Coimbra } \\
\hline Bezerra, George & \\
\hline Ferreira, Adelino & \\
\hline Alho, Andre Romano & University of Lisbon \\
\hline Oliveira, Jose Fernando & \multirow[t]{2}{*}{ University of Porto } \\
\hline Rosa-Santos, Paulo & \\
\hline \multicolumn{2}{|c|}{ Romania } \\
\hline $\begin{array}{l}\text { Bodea, Constanta- } \\
\text { Nicoleta }\end{array}$ & $\begin{array}{l}\text { Bucharest University } \\
\text { of Economic Studies }\end{array}$ \\
\hline Burnete, Nicolae & \multirow{2}{*}{$\begin{array}{l}\text { Technical University } \\
\text { of Cluj-Napoca }\end{array}$} \\
\hline Mariasiu, Florin & \\
\hline
\end{tabular}

\begin{tabular}{|c|c|}
\hline Surname, Name & Institution \\
\hline \multicolumn{2}{|c|}{ Russian Federation } \\
\hline Kulmanakov, Sergey & Altai State Technical University \\
\hline Koryagin, Mark & $\begin{array}{l}\text { Kemerovo State Agricultural } \\
\text { Institute }\end{array}$ \\
\hline Andreev, Vladimir & $\begin{array}{l}\text { Moscow State University } \\
\text { of Civil Engineering }\end{array}$ \\
\hline Dyakov, Ivan & $\begin{array}{l}\text { Ulyanovsk State Technical } \\
\text { University }\end{array}$ \\
\hline \multicolumn{2}{|r|}{ Serbia } \\
\hline Stanujkić, Dragiša & Megatrend University \\
\hline Babić, Obrad & \multirow[t]{10}{*}{ University of Belgrade } \\
\hline Bojović, Nebojša & \\
\hline Čokorilo, Olja & \\
\hline Jović, Jadranka & \\
\hline Milenković, Miloš & \\
\hline Netjasov, Fedja & \\
\hline Pešić, Dalibor & \\
\hline Radmilović, Zoran & \\
\hline Rusov, Srđan & \\
\hline Vujanovic, Davor & \\
\hline Petrović, Dragan & University of Novi Sad \\
\hline \multicolumn{2}{|c|}{ Singapore } \\
\hline Choor, Chin Hoong & \multirow{4}{*}{$\begin{array}{l}\text { National University } \\
\text { of Singapore }\end{array}$} \\
\hline Fang, Fwa Tien & \\
\hline Peng, Kemao & \\
\hline $\mathrm{Xu}$, Jianxin & \\
\hline Wang, David & $\begin{array}{l}\text { Nanyang Technological } \\
\text { University }\end{array}$ \\
\hline Wilhelm, Erik & $\begin{array}{l}\text { Singapore University } \\
\text { of Technology and Design }\end{array}$ \\
\hline \multicolumn{2}{|r|}{ Slovakia } \\
\hline Adamčik, František & \multirow[t]{2}{*}{ Technical University of Košice } \\
\hline Guzanová, Anna & \\
\hline Adamko, Norbert & \multirow[t]{9}{*}{ University of Žilina } \\
\hline Barta, Dalibor & \\
\hline Bugaj, Martin & \\
\hline Gašparík, Jozef & \\
\hline Gutten, Miroslav & \\
\hline Havel, Karel & \\
\hline Kasanický, Gustáv & \\
\hline Koháni, Michal & \\
\hline Matis, Peter & \\
\hline
\end{tabular}




\begin{tabular}{|c|c|}
\hline Surname, Name & Institution \\
\hline \multicolumn{2}{|c|}{ Slovenia } \\
\hline Bajec, Patricija & \multirow[t]{6}{*}{ University of Ljubljana } \\
\hline Batista, Milan & \\
\hline Beškovnik, Bojan & \\
\hline Šelih, Jana & \\
\hline Twrdy, Elen & \\
\hline Žura, Marijan & \\
\hline Klanšek, Uroš & \multirow[t]{2}{*}{ University of Maribor } \\
\hline Tollazzi, Tomaž & \\
\hline \multicolumn{2}{|r|}{ Spain } \\
\hline Godoy, Jorge & $\begin{array}{l}\text { Centre for Automation and } \\
\text { Robotics, AUTOPIA }\end{array}$ \\
\hline Onieva, Enrique & Deusto Institute of Technology \\
\hline Casal, Joaquim & $\begin{array}{l}\text { Polytechnic University of } \\
\text { Catalonia }\end{array}$ \\
\hline Cabrera, Suitberto & $\begin{array}{l}\text { Polytechnic University of } \\
\text { Valencia }\end{array}$ \\
\hline Ayuga, Francisco & \multirow[t]{3}{*}{ Technical University of Madrid } \\
\hline Castro, Maria & \\
\hline Gallego, Eutiquio & \\
\hline Fernández-Llorca, David & University of Alcalá \\
\hline Alonso, Borja & \multirow[t]{2}{*}{ University of Cantabria } \\
\hline Dell‘Olio, Luigi & \\
\hline Calvo, Francisco & \multirow[t]{4}{*}{ University of Granada } \\
\hline De Oña, Rocío & \\
\hline Di Stasi, Leandro Luigi & \\
\hline Garach, Laura & \\
\hline Veldman, Simme & University of Oviedo \\
\hline Munuzuri, Jesus & University of Seville \\
\hline Cantos, Pedro & University of Valencia \\
\hline \multicolumn{2}{|c|}{ Sweden } \\
\hline Forsman, Åsa & \multirow{3}{*}{$\begin{array}{l}\text { Swedish National Road and } \\
\text { Transport Research Institute }\end{array}$} \\
\hline Kharrazi, Sogol & \\
\hline Linder, Astrid & \\
\hline Olsson, Jerry & University of Gothenburg \\
\hline \multicolumn{2}{|c|}{ Switzerland } \\
\hline Onder, Christopher & \multirow{2}{*}{$\begin{array}{l}\text { Swiss Federal Institute of } \\
\text { Technology in Zurich }\end{array}$} \\
\hline Vilkelis, Aurimas & \\
\hline
\end{tabular}

\begin{tabular}{|c|c|}
\hline Surname, Name & Institution \\
\hline \multicolumn{2}{|r|}{ Taiwan } \\
\hline Hsu, Hui-Mi & National Ilan University \\
\hline Lin, Cherng-Yuan & $\begin{array}{l}\text { National Taiwan Ocean } \\
\text { University }\end{array}$ \\
\hline Yu, Vincent & $\begin{array}{l}\text { National Taiwan University of } \\
\text { Science and Technology }\end{array}$ \\
\hline \multicolumn{2}{|r|}{ Turkey } \\
\hline Çelik, Ali Kemal & Atatürk University \\
\hline $\begin{array}{l}\text { Rouyendegh, Babak } \\
\text { Daneshvar }\end{array}$ & Atılım University \\
\hline Tanyel, Serhan & Dokuz Eylül University \\
\hline Demirel, Hande & \multirow[t]{3}{*}{ Istanbul Technical University } \\
\hline Kaya, Tolga & \\
\hline Turk, Sevkiye Sence & \\
\hline Bezgin, Niyazi Özgür & \multirow[t]{2}{*}{ Istanbul University } \\
\hline Karaşahin, Mustafa & \\
\hline Akgüngör, Ali & Kırıkkale University \\
\hline Ceylan, Halim & \multirow[t]{2}{*}{ Pamukkale University } \\
\hline Ceylan, Huseyin & \\
\hline Akbıyıklı, Rifat & \multirow[t]{2}{*}{ Sakarya University } \\
\hline Güler, Hakan & \\
\hline \multicolumn{2}{|r|}{ Ukraine } \\
\hline Perig, Olexandr & $\begin{array}{l}\text { Donbass State Engineering } \\
\text { Academy }\end{array}$ \\
\hline Bieliatynskyi, Andrii & \multirow[t]{5}{*}{ National Aviation University } \\
\hline Glazunov, Nikolaj & \\
\hline Grekhov, Andrii & \\
\hline Ivannikova, Viktoriia & \\
\hline Krayushkina, Kateryna & \\
\hline Klymenko, Iryna & $\begin{array}{l}\text { National University of } \\
\text { Transport }\end{array}$ \\
\hline Maruschak, Pavlo & $\begin{array}{l}\text { Ternopil Ivan Pul'uj National } \\
\text { Technical University }\end{array}$ \\
\hline \multicolumn{2}{|c|}{ United Arab Emirates } \\
\hline Ahmed, Kamran & $\begin{array}{l}\text { United Arab Emirates } \\
\text { University }\end{array}$ \\
\hline \multicolumn{2}{|c|}{ United Kingdom of Great Britain and Northern Ireland } \\
\hline Mason, Robert & \multirow[t]{2}{*}{ Cardiff University } \\
\hline Nair, Rawindaran & \\
\hline Velenis, Efstathios & Cranfield University \\
\hline
\end{tabular}




\begin{tabular}{|c|c|}
\hline Surname, Name & Institution \\
\hline Carreno, Michael & \multirow[t]{5}{*}{ Edinburgh Napier University } \\
\hline Grieco, Margaret & \\
\hline Kumar, Ravindra & \\
\hline Saleh, Wafaa & \\
\hline Stewart, Kathryn & \\
\hline Connolly, David & Heriot-Watt University \\
\hline Angeloudis, Panagiotis & \multirow[t]{3}{*}{ Imperial College London } \\
\hline $\mathrm{Han}, \mathrm{Ke}$ & \\
\hline Krishnan, Rajesh & \\
\hline Volkov, Konstantin & Kingston University \\
\hline Eglese, Richard & $\begin{array}{l}\text { Lancaster University } \\
\text { Management School }\end{array}$ \\
\hline Enoch, Marcus & \multirow[t]{5}{*}{ Loughborough University } \\
\hline Li, Baibing & \\
\hline Lombardo, Teresa & \\
\hline Pitfield, David & \\
\hline Ward, Christopher & \\
\hline Aditjandra, Paulus & \multirow[t]{2}{*}{ Newcastle University } \\
\hline Galatioto, Fabio & \\
\hline Corsar, David & \multirow[t]{3}{*}{ University of Aberdeen } \\
\hline Cottrill, Caitlin & \\
\hline Nelson, John & \\
\hline Erdoğan, Güneş & University of Bath \\
\hline Kaewunruen, Sakdirat & \multirow[t]{2}{*}{ University of Birmingham } \\
\hline Schmid, Felix & \\
\hline Kashani, Mehdi & \multirow[t]{4}{*}{ University of Bristol } \\
\hline Shterenlikht, Anton & \\
\hline Wanous, Mohammed & \\
\hline Zhang, Jie & \\
\hline Kinuthia, John & University of Glamorgan \\
\hline Ropkins, Karl & University of Leeds \\
\hline Konstantopoulos, Panos & University of Nottingham \\
\hline Cherrett, Tom & University of Southampton \\
\hline Blair, Neale & University of Ulster \\
\hline \multicolumn{2}{|c|}{ United States } \\
\hline Zhou, Xuesong & Arizona State University \\
\hline Baratian, Fatemeh & Auburn University \\
\hline Wei, Junqing & Carnegie Mellon University \\
\hline Xiong, Qingang & FCA US LLC \\
\hline Cornillie, Thomas & Independent Scholar \\
\hline
\end{tabular}

\begin{tabular}{|c|c|}
\hline Surname, Name & Institution \\
\hline Ji, Yigong & $\begin{array}{l}\text { Indiana Department } \\
\text { of Transportation }\end{array}$ \\
\hline Dong, Jing & \multirow[t]{5}{*}{ Iowa State University } \\
\hline $\begin{array}{l}\text { Gopalakrishnan, } \\
\text { Kasthurirangan }\end{array}$ & \\
\hline Sharma, Anuj & \\
\hline Smadi, Omar & \\
\hline Santhanam, Ganesh Ram & \\
\hline Daphalapurkar, Nitin & Johns Hopkins University \\
\hline Bullough, John & $\begin{array}{l}\text { Lighting Research Center, } \\
\text { Rensselaer Polytechnic Institute }\end{array}$ \\
\hline Xiao, Danny & $\begin{array}{l}\text { Louisiana Transportation } \\
\text { Research Center }\end{array}$ \\
\hline Zoepf, Stephen & $\begin{array}{l}\text { Massachusetts Institute } \\
\text { of Technology }\end{array}$ \\
\hline Hu, Xianbiao & Metropia Inc. \\
\hline Serpa, Jose & Miami Dade College \\
\hline Lautala, Pasi & \multirow{3}{*}{$\begin{array}{l}\text { Michigan Technological } \\
\text { University }\end{array}$} \\
\hline Lidicker, Jeffrey & \\
\hline Pouryousef, Hamed & \\
\hline Newman, James & Mississippi State University \\
\hline Yager, Thomas & $\begin{array}{l}\text { National Aeronautics and Space } \\
\text { Administration (NASA) }\end{array}$ \\
\hline Agrawal, Ankit & Northwestern University \\
\hline Ye, Fan & Ohio Northern University \\
\hline Jeon, Christy & Parsons Corporation \\
\hline Donnell, Eric & Pennsylvania State University \\
\hline Brownell, Chris & Princeton University \\
\hline $\begin{array}{l}\text { Yadollahi, } \\
\text { Mohammadreza }\end{array}$ & Rowan University \\
\hline Deka, Devajyoti & \multirow[t]{3}{*}{ Rudgers University } \\
\hline Shi, Jin & \\
\hline Yi, Jingang & \\
\hline Liu, Litao & $\begin{array}{l}\text { Texas A\&M Transportation } \\
\text { Institute }\end{array}$ \\
\hline Duru, Okan & $\begin{array}{l}\text { Texas A\&M University } \\
\text { at Galveston }\end{array}$ \\
\hline Yang, Xianfeng & University of Maryland \\
\hline Shaheen, Susan & \multirow{2}{*}{$\begin{array}{l}\text { University of California } \\
\text { Berkeley }\end{array}$} \\
\hline Wei, Dali & \\
\hline Oum, Sanghyouk & University of California Davis \\
\hline Garrick, Norman & University of Connecticut \\
\hline James, Leon & University of Hawai'i System \\
\hline
\end{tabular}




\begin{tabular}{|c|c|}
\hline Surname, Name & Institution \\
\hline Zhao, Ming & University of Houston \\
\hline Fitzsimmons, Eric & University of Kansas \\
\hline Gårder, Per & University of Maine \\
\hline Golias, Mihalis & University of Memphis \\
\hline Mudgal, Abhisek & University of Minnesota \\
\hline Hajj, Elie & University of Nevada, Reno \\
\hline Dong, Chunjiao & University of Tennessee \\
\hline Zhou, Qian & University of Virginia \\
\hline Chitturi, Madhav & \multirow{2}{*}{$\begin{array}{l}\text { University of Wisconsin- } \\
\text { Madison }\end{array}$} \\
\hline Li, Zhixia & \\
\hline \multicolumn{2}{|c|}{ Vietnam } \\
\hline Viet, La Duc & $\begin{array}{l}\text { Institute of Mechanics, Vietnam } \\
\text { Academy of Science and } \\
\text { Technology }\end{array}$ \\
\hline
\end{tabular}

